\title{
Highs and Lows of Proactive Therapeutic Drug Monitoring in Crohn's Patients
}

\author{
Shana T. Rakowsky ${ }^{1}$ Joseph D. Feuerstein ${ }^{1}$
}

Accepted: 7 December 2020 / Published online: 12 January 2021

(c) The Author(s), under exclusive licence to Springer Science+Business Media, LLC part of Springer Nature 2021

Therapeutic drug monitoring (TDM) has emerged in the field of inflammatory bowel disease (IBD) as an important clinical tool aimed at optimizing the patient's response to biologic therapy. TDM involves measuring serum concentrations of a therapeutic drug or antidrug antibodies with the aim of inducing (reactive) or sustaining (proactive) disease remission through confirming that drug dosing is adequate. Its rising importance has been supported by studies correlating reactive TDM measurements and favorable patient outcomes, including decreased hospitalizations, surgery, colectomy, and loss of response [1]. While less robust, studies have also shown that proactive TDM similarly provides improved clinical outcomes, reducing loss of response, and providing a cost-effective method for dosing biologics [2].

Nevertheless, given the relative novelty of TDM and gaps in the literature, there continues to be uncertainty regarding the logistics of how and when to apply TDM into clinical practice [3]. Unresolved questions include how should TDM be performed-proactive monitoring vs reactive monitoring, how often should proactive testing be performed, which patients benefit most from TDM, how to interpret and respond to the results, and which are the most appropriate concentrations to target. The American Gastroenterological Association (AGA) guideline on TDM suggests the use of reactive testing though noting the quality of evidence is very low [4]. In contrast, the data supporting proactive monitoring remains challenging given the limited number of randomized control trials. Most studies are retrospective with concerns regarding bias in study design that may exaggerate the efficacy of proactive TDM. Nonetheless,

Joseph D. Feuerstein

jfeuerst@bidmc.harvard.edu

Shana T. Rakowsky

srakowsk@bidmc.harvard.edu

1 Division of Gastroenterology, Department of Medicine, Beth Israel Deaconess Medical Center, 110 Francis St 8e Gastroenterology, Boston, MA 02215, USA the challenge remains, when and how often should proactive TDM be performed and most importantly though, if proactive TDM reduces the occurrence of future negative outcomes that are relevant to patients-i.e., drug failure, surgery, and hospitalization.

The study by Borren et al. published in this issue of Digestive Diseases and Sciences [5] sought to help address some of these questions by studying the effects of TDM in patients with quiescent IBD receiving infliximab maintenance therapy. Patients were confirmed to be in clinical and endoscopic remission. Infliximab concentrations were obtained at the time of study enrollment when patients were in remission. Patients were monitored over the next two years for evidence of clinical disease relapse, with the study concluding that infliximab trough concentrations obtained during periods of remission did not predict future relapse. They also noted that baseline infliximab concentrations correlated strongly with levels at 12 and 24 months, validating the stability of serum concentrations in quiescent disease. The lack of future predictive value of TDM on disease relapse and the relative stability of the drug levels questions the value of proactive TDM in patients with quiescent disease who have been stable on infliximab regimens.

Using the data from this study, one may consider opting against proactively monitoring infliximab concentrations given the lack of improved outcomes. However, opting against proactive monitoring can feel unsettling, as it seems to contradict the underlying concept of TDM-that biologic drugs require a set concentration in order to be therapeutic. By not checking and not knowing the concentration, there is a risk of underdosing the medication with a theoretical risk of loss of response. The literature suggests that up to one-third of patients with IBD show primary non-response to biologic therapies and up to $50 \%$ of patients after initial clinical response stop therapy for either secondary loss of response or a serious adverse advent [6]. Moreover, patient-specific factors may also alter drug concentrations: body weight, serum albumin, disease subtype, 
gender, and frequency of bowel movements [1]. Nevertheless, adequate tools that predict how infliximab concentrations may change in individual patients over time are not yet available. Moreover, and of concern, it is not possible to predict which patients will respond to which biologic therapies. Borren et al. [5] reported stable infliximab concentrations over time in their patient population, albeit data were only available for a limited percentage of their patient population. The stability was posited to be due to less fecal loss of drug in quiescent disease. Nonetheless, is the presumption of the long-term stability of drug concentrations valid? The patient's weight may change, dosing intervals may be inadvertently altered, or diarrheal illnesses might increase drug excretion-all potentially lowering the drug concentration. While this study does suggest that stable patients with quiescent disease should remain adequately dosed and remain in remission, clinicians still need to be monitoring patients for conditions that would lower drug concentrations over time.

The findings of this study also challenge one to consider another question, namely what is the harm in monitoring infliximab concentrations in patients with quiescent disease? As stated above, these patients are already engaged with regular office visits and laboratory monitoring. Costs incurred by patients and the healthcare system as a result of unnecessary testing are certainly an important factor to consider, though this must be balanced with the physical and financial risks associated with a flare or need for change in therapy due to loss of response. Yet, most of this concern for the loss of response during maintenance therapy in remission remains theoretical. Though the ideal trough concentration during remission is often reported as $\geq 5 \mu \mathrm{g} / \mathrm{mL}$, as noted in the AGA technical review, most patients will be in remission even with levels as low as $3 \mu \mathrm{g} / \mathrm{mL}$ with even fewer patients failing to respond when higher levels are targeted [1]. The need to optimize a level when someone is in remission, however, remains unclear. The study by Borren et al. [5] suggests that this level is likely to remain stable and does not predict the risk of flaring. In fact, their study showed no difference in trough levels in those who relapsed versus those who did not $(7.5 \mu \mathrm{g} / \mathrm{mL} \pm 3.7 \mu \mathrm{g} / \mathrm{mL}$ vs. $8.1 \mu \mathrm{g} / \mathrm{mL} \pm 7.9 \mu \mathrm{g} / \mathrm{mL}$, $p=0.815$ ) even though levels were typically above the target $5 \mu \mathrm{g} / \mathrm{mL}$ [5]. This begs the fundamental question - is TDM needed for patients in remission?

Overall, the study successfully brings to the forefront the importance of ongoing investigation into proactive TDM.
Given the complex nature of caring for patients with IBD with our expanding, but still limited, therapeutic armamentarium, a better understanding of available tools such as TDM is crucial to providing the highest quality care. Additional research focused on the optimal use of TDM, including when to use it and on whom to use it will help facilitate necessary movement toward standardization of TDM in clinical practice.

\section{Compliance with Ethical Standards}

Conflict of interest Both authors declare that they have no conflict of interest.

\section{References}

1. Casteele NV, Herfarth H, Katz J, Falck-Ytter Y, Singh S. American Gastroenterological Association Institute technical review on the role of therapeutic drug monitoring in the management of inflammatory bowel diseases. Gastroenterology 2017;153:835-857

2. Roblin X, Riviere P, Flamant $M$ et al. Proactive therapeutic drug monitoring of TNF antagonists in inflammatory bowel disease. Inflamm Bowel Dis 2018;24:1904-1909

3. Shah R, Hoffman GR, El-Dallal M, Goldowsky AM, Chen Y, Feuerstein JD. Is therapeutic drug monitoring for anti-tumour necrosis factor agents in adults with inflammatory bowel disease ready for standard of care? A systematic review and meta-analysis. J Crohn's Colitis 2020;14:1057-1064.

4. Feuerstein J, Nguyen G, Kupfer S, Falck-Ytter Y, Singh S. American gastroenterological association institute clinical guidelines committee. American Gastroenterological Association Institute Guideline on Therapeutic Drug Monitoring in inflammatory bowel disease. Gastroenterology 2017;153:827-34.

5. Borren, NZ, Paulides, E, Frinack, JL et al. Infliximab trough levels are not predictive of relapse in patients with IBD in endoscopic remission: a multicenter cohort study. Dig Dis Sci. (Epub ahead of print). https://doi.org/10.1007/s10620-020-06645-0.

6. Papamichael K, Cheifetz AS, Melmed GY et al. Appropriate therapeutic drug monitoring of biologic agents for patients with inflammatory bowel diseases. Clin Gastroenterol Hepatol 2019; $17: 1655-1668$

Publisher's Note Springer Nature remains neutral with regard to jurisdictional claims in published maps and institutional affiliations. 